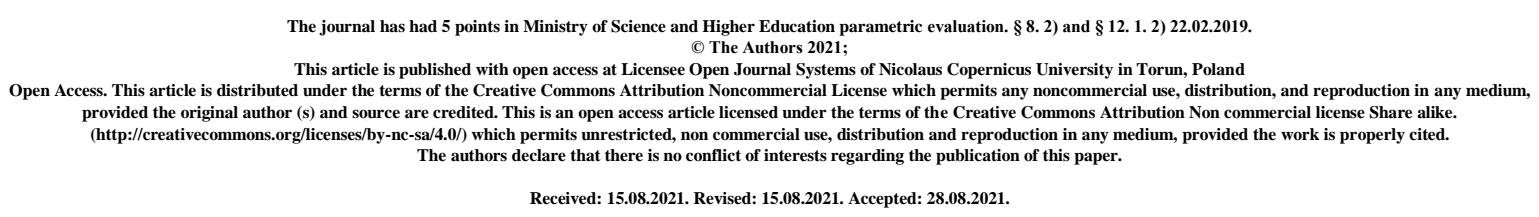

\title{
Selected skin diseases of the feet
}

\section{Wiktoria Chodun ${ }^{1}$, Kinga Ruszel ${ }^{1}$, Robert Dubel ${ }^{1}$, Barbara Nieradko-Iwanicka ${ }^{2}$}

${ }^{1}$ Students Scientific Association at the Chair and Department of Hygiene Medical University of Lublin

${ }^{2}$ Chair and Department of Hygiene, Medical University of Lublin Radziwiłowska 11 Street, 20080 Lublin

Correspondence: Wiktoria Chodun

e-mail wiktoria.chodun19@wp.pl

\begin{abstract}
Foot skin diseases are common problems encountered by dermatologists. They involve the skin and nails. In addition to the health aspect, there is also an aesthetic aspect, especially troublesome in the summer. It is recommended to visit a dermatologist with any disturbing skin lesion. The article presents an overview of the most common diseases in dermatology and podiatry offices.
\end{abstract}

Key words: athlete's foot, calluses, warts, mycoses 


\section{Introduction}

Foot skin diseases are common problems encountered by dermatologists. They involve the skin and nails. In addition to the health aspect, there is also an aesthetic aspect, especially troublesome in the summer. It is recommended to visit a dermatologist with any disturbing skin lesion.

\section{Methods}

The article is a review of 5 publications on feet skin diseases.

Results and Discussion

\section{Mycoses}

Mycoses of the feet are caused by fungi, most often dermatophytes such as Trichophyton rubrum and Trichophyton mentagrophytes var. Interdigitale. Interdigital mycosis is the most common clinical variety. The lesions occupy the interdigital spaces, starting from the skin between the 4th and 5th toes, and as the disease gets worse, it may also spread to the surrounding skin. The changes are maceration and exfoliation of the epidermis in the depths of the folds, inflammatory erythema, sometimes with the presence of cracks and oozing erosions. The smallest inflammatory reaction is caused by dermatophytes specialized in attacking humans anthropophilic, which is associated with a more chronic, sometimes long-term nature of infection. When spreading the infection to the back of the foot, mycoses can be recognized by appearance, there is an active edge, erythema and papules with intensification around the perimeter, sometimes vesicles and pustules. Itching of the skin varies in severity - from complete absence to moderate, but usually less than in eczema-like diseases. The pain is caused by erosions and cracks. The course of the disease is chronic with periodic exacerbations [1].

Exfoliating mycosis is called moccasin because of its characteristic location. The changes occurring on the feet take the form of confluent, dry erythematous-exfoliating foci. It affects the soles of the feet, the lateral surfaces and heels, and partially the back of the foot, mainly in the toes. The erythema is better visible on the dorsal side, only increased keratosis and exfoliation may be visible in the soles of the feet. There is little or no itching. The course of the disease, as in the case of interdigital mycosis, is chronic[2].

Another type of mycosis is athlete's foot (dyshydrotic). The changes only affect the plantar side of the feet. Periodically, once every few weeks or months, numerous small blisters are sown on the erythematous substrate or larger. Originally with serous content and severe itching. Over time, the blisters burst with erosion and peeling. The presence of erosions causes burning and soreness. The changes disappear gradually, even if left untreated, but after some time the disease comes back[3].

The diagnosis of mycoses is based on a mycological examination. In the treatment, antifungal agents of moccasin mycosis are used, and local treatment can be used. It is impossible to forget about the succession of disinfection, but also socks and ales, without which there is a high risk of relapse. Predisposing factors are, among others, wet shoes, the use of someone else's shoes, excessive sweating, diabetes, old age, not practicing hygiene in saunas or swimming pools[3].

Viral warts 
Warts are caused by the human papilloma viruses (HPVs). The infection occurs in direct contact. Foot warts are hyperkeratotic lumps, usually skin-colored or slightly lighter, a few to several millimeters in size, with a warty surface, sometimes with characteristic black points, which are blood microclots in overgrown loops of capillaries. They can be single or multiple, located both on the soles and on the backs of the feet, toes, in particular on the nail shafts. Usually they do not cause subjective symptoms, however large warts on the sole of the foot and periungual warts can be painful. They are much more common in children than in adults and are often associated with early school age or visits to the swimming pool. Chronic course, but most resolves spontaneously within a few years. One of the most popular ways to deal with warts is to exfoliate the epidermis with salicylic, lactic or trichloroacetic acids. A variety of topical treatments are used in treatment, including keratolytic preparations and cryodestruction. The presence of the virus in the apparently healthy epidermis can cause warts to come back quickly after treatment. Studies show that combined acid therapy and nipple freezing shorten treatment time. When this treatment fails, the cytostatic 5-fluorouracil can be used. It inhibits the growth of the virus and infected cells. For supporters of natural medicine, celandine will be helpful in combating warts. Enzymes contained in it soften the skin, thus improving the absorption of compounds that inhibit cell proliferation. The most effective is the juice of a freshly picked plant, which is applied to the wart several times a day, avoiding the area of healthy tissue. There are two types of foot warts. Myrmecia warts - caused by HPV 1 viruses are large, solitary, painful, penetrating deeply into the skin and causing inflammation, they usually disappear permanently and require differentiation from corns (corns). HPV 2-related mosaic warts - The same virus that causes common warts on the hands, they are superficial, small, numerous, usually painless, and often recur after resolution[1,2,3].

Corns and calluses

Calluses are poorly delimited, hyperkeratosis foci of various sizes. They do not cause pain, they arise as a result of chronic pressure or friction of the skin, most often as a result of wearing tight shoes or deformation of the feet or toes, which causes uneven distribution of the load. Calluses are usually yellowish in color, the skin becomes convex, and can also be rough or raised. They should be differentiated from fingerprints[1,2,3].

\section{Corns}

Corns are several millimeters high hyperkeratotic thickening with the corneal core in the central part, which, by pressing on soft tissues, causes pain. They are more demarcated from the skin than calluses. They occur practically only on the feet within the soles, often also in the interdigital spaces. The cause of corns is incorrectly selected footwear, i.e. too small, too narrow. They are often found in dancers and athletes. The course of the disease is extremely chronic. The treatment uses keratolytic preparations, cryodestruction, mechanical abrasion, and surgical removal. If the cause of the corn formation is not removed, the changes will return[1,2,3].

\section{Psoriasis of the feet}

Psoriasis of the feet is a form of ordinary psoriasis. It is an autoimmune disease. The primary bloom is a reddish-brown lump covered with a silvery scales. Characteristic and helpful in the diagnosis of the disease is the Köbner's symptom, i.e. the formation of psoriatic lesions on the skin in an active disease for about 8-14 days, even a minor local trauma to the epidermis. The 
papules tend to widen and merge peripherally. This symptom is often called the stearin candle symptom. Plaque psoriasis most often affects the knees, elbows, the sacrum area and the scalp. Psoriasis vulgaris of the hands and feet is a very rare clinical form of psoriasis vulgaris with the appearance of psoriatic papules on the palms and soles of the feet, due to the beneficial effect of exposure to sunlight means that lesions do not usually form on the exposed skin. Different diseases must be considered in differentiation, including ringworm, eczema, lichen planus and recurrent syphilis. The diagnosis is made on the basis of the clinical picture, and in doubtful cases, the histological examination is decisive. Treatment is local and includes anthralin and corticosteroids, phototherapy, and in severe, resistant cases generally methotrexate or cyclosporine A $[1,2,3,4]$.

\section{Lichen planus}

Lichen planus is probably an autoimmune condition. The classic form is characterized by the presence of changes on the skin of polygonal flat lumps, pink to dark burgundy or dark purple in color, glistening in the side light, with a tendency to focus. As in psoriasis, there may be a Köbner symptom. The skin lesions are usually very itchy. They are located symmetrically, mainly within the ankle joints of the feet. The changes disappear while leaving the discoloration. There may also be changes in the nails, the most typical is longitudinal grooving. In the overgrown variety, the overgrown papules with a hyperkeratotic surface merge into larger foci covered with abundant scales, blurring the structure of individual papules. The lesions are very itchy and are located on the lower legs and backs of the feet. Patients complaining of itching, however, most often rub the skin without scratching, for fear of the appearance of further lesions. The clinical picture, not resembling lichen planus, requires differentiation from corns, calluses, viral warts, exfoliative mycosis and keratotic eczema. In doubtful cases, the diagnosis of lichen planus is determined by the histopathological and immunofluorescence examination of the lesions. Treatment includes topical and systemic corticosteroids, other immunosuppressants, retinoids, and phototherapy[1,2,3].

\section{Erythema multiforme exudative}

It is a reaction of the immune system in response to viral infections (e.g. influenza or upper respiratory tract infections, herpes simplex labial), bacterial (streptococci) and medication (antibiotics, anticonvulsants, antimalarials, sulfonamides, non-steroidal anti-inflammatory drugs, diuretics). The reasons are not fully known. Erythema occurs in both women, men and children. There are several clinical varieties within the disease entity, differing in the severity of the course: mild (smaller) form with disseminated skin lesions only, mainly on distant parts of the limbs, severe (larger) form with accompanying changes on the mucous membranes, Stevens-Johnson syndrome with the dominant involvement of mucous membranes and skin lesions mainly on the trunk and face, toxic necrolysis of the Lyell's epidermis, where the skin develops confluent erythema and epidermis creeping, as in second-degree burns, on more than $30 \%$ of the body surface. Diagnosis of exudative erythema multiforme is based on the clinical picture. The disease begins suddenly with fever, muscle and joint pains, which in mild form disappear after a few days, while skin changes persist for several weeks. The milder form of exudative erythema multiforme occurs in the form of erythematous-edema lesions of varying severity of inflammation. The affected areas of the skin are symmetrical and take the form of concentric shapes and rings. It happens that blisters and hemorrhagic changes appear. The location is symmetrical, mainly on the weakened parts of the limbs, on both sides of the hands 
and feet. Many changes often disappear with massive exfoliation of the epidermis from the entire surface of the hands and feet ("socks and gloves" exfoliation). They are mainly located in the mouth and lips. The erosions are covered with characteristic hemorrhagic scabs. Wounds should be properly cared for to prevent infections. Treatment depends on the severity of the disease and the causative agent [5].

\section{Summary}

Foot skin diseases should not be underestimated. The sooner help is provided and treatment is selected, the faster the health and aesthetic condition will improve. Preventive measures to avoid foot diseases include not borrowing shoes, thoroughly drying feet after bathing, maintaining hygiene in swimming pools and in the sauna, especially in the case of diseases such as diabetes, following the doctor's instructions and careful monitoring of the condition of the feet.

\section{Bibliography}

1.Nowicka D. Dermatologia, Górnicki Wydawnictwo Medyczne, Wrocław 2007.

2.Lauritz B. Dermatoses of the feet, Am J Clin Dermatol., 2000;1: 181-186.

3. Adamski Z., Kaszuba A. (Ed.) Dermatologia dla kosmetologów, Wydawnictwo Naukowe Uniwersytetu Medycznego im. Karola Marcinkowskiego, Poznań 2008

4. Żelazny I., Nowicki R., Majkowic M., Samet M. Jakość życia w chorobach skóry, Przewodnik

Lekarza, 2004;9: 60-65.

5. Huff J. C., Weston W. L., Tonnesen M. G. Erythema multiforme: a critical review of characteristics, diagnostic criteria, and causes. Journal of the American Academy of Dermatology. 1983;8(6):763-775. doi: 10.1016/S0190-9622(83)80003-6 